\title{
DISPERSION-FREE STATES AND THE EXCLUSION OF HIDDEN VARIABLES
}

\section{S. P. GUDDER}

1. Introduction. John von Neumann [8] proved his famous "hidden variables" theorem by showing that a coherent quantum mechanical system has no dispersion-free states. In this proof von Neumann assumed that the quantum mechanical logic $L$ consisted of all closed subspaces of an infinite dimensional Hilbert space. There has been great interest lately in more general quantum mechanical logics and recently Jauch and Piron [4] extended von Neumann's result to the case in which $L$ is a complete complemented lattice. In this note we generalize and sharpen this result by assuming that $L$ is an orthocomplemented partially ordered set which is complete with respect to compatible elements. The importance of this generalization physically lies in the fact that there seems to be no experimental evidence that $L$ should be complete with respect to arbitrary elements, although completeness with respect to compatible elements is physically much more reasonable; and further, doubts have been raised that $L$ should even be a lattice (cf. [7]). In this note we prove two theorems; the first characterizes quantum mechanical logics which have a dispersion-free state, and the second characterizes quantum mechanical logics which admit hidden variables.

2. Definitions. We give an axiomatic formulation of quantum mechamics which is equivalent to that of Jauch and Piron [4] except it is weakened to the extent mentioned in $\$ 1$. Let $L=\{a, b, c, \cdots\}$ be a partially ordered set with first and last elements 0,1 respectively and a complementation $a \rightarrow a^{\prime}$ which satisfies $\left(a^{\prime}\right)^{\prime}=a, a \leqq b$ implies $b^{\prime} \leqq a^{\prime}$, and $a \bigvee a^{\prime}$ exists and is 1 for all $a$ in $L$. We call the elements of $L$ propositions. We say that $a$ and $b$ are disjoint (written $a \perp b$ ) if $a \leqq b^{\prime}$ and we write $a+b$ for $a \bigvee b$ if $a \perp b$. We say that $a$ and $b$ are compatible (written $a \leftrightarrow b$ ) if there are mutually disjoint propositions $a_{1}, b_{1}, c$ such that $a=a_{1}+c, b=b_{1}+c$. If $a \leqq b$ we assume that there is a $c$ in $L$ such that $a+c=b$. This corresponds to Axiom $\mathrm{P}$ in [4]. We call $L$ a $\operatorname{logic}$ if for every collection $\left\{a_{\alpha}: \alpha \in A\right\}$ of mutually compatible propositions $\vee a_{\alpha}, \wedge a_{\alpha}$ exists.

A state $m$ on $L$ is a real valued function on $L$ satisfying $0 \leqq m(a)$ for every $a$ in $L, m(1)=1$, and $m(a+b)=m(a)+m(b)$. If $m_{i}, i=1,2, \cdots$ is a sequence of states and $\lambda_{i} \geqq 0, i=1,2, \cdots, \sum \lambda_{i}=1$ we define

Received by the editors August 18, 1966. 
the state $m=\sum \lambda_{i} m_{i}$ by $m(a)=\sum \lambda_{i} m_{i}(a)$ for every $a$ in $L$. We call $m$ a mixture of the states $m_{i}$. The pair $(L, M)$ is called a quantum system if $L$ is a logic and $M$ a set of states on $L$ which is closed under mixtures and satisfies:

(M1) if $a \neq 0$ there is an $m$ in $M$ such that $m(a)=1$;

(M2) if $m(a)=m(b)=1, m \in M$, and if $a \wedge b$ exists then $m(a \wedge b)=1$;

(M3) if $\left\{a_{\alpha}: \alpha \in A\right\}$ are mutually compatible and $m\left(a_{\alpha}\right)=1$, $\alpha \in A, m \in M$, then $m\left(\bigwedge a_{\alpha}\right)=1$.

Notice that (M2) corresponds to (4) ${ }^{\circ}$ and (M3) is a much weakened version of (4) in [4]. In $\$ 5$ we shall give an example of a quantum system which is not a lattice, and hence our axioms are strictly weaker than those in [4].

The center $Z$ of $L$ is the set of propositions which are compatible with every proposition. $L$ is coherent, deterministic, or trivial if $Z=\{0,1\}, Z=L$, or $L=\{0,1\}$ respectively. We say that $(L, M)$ is coherent, deterministic, or trivial if $L$ is. Note that $L$ is a Boolean algebra if and only if it is deterministic. A proposition $a$ is an atom if $a \neq 0$ and if $b \leqq a$ implies $b$ is $a$ or $0 . L$ is atomic if every nonzero proposition contains an atom. A state $m$ is dispersion-free if $m$ has only the values 0 and 1. A logic $L$ admits hidden variables if there is a set of states $M$ which are mixtures of dispersion-free states such that $(L, M)$ is a quantum system.

We now give a lemma which will simplify our work to some extent. Its proof is routine.

Lemma. A logic L admits hidden variables if and only if there is a set $M$ of dispersion-free states which satisfies (M1), (M2), and (M3).

\section{Dispersion-free states and hidden variables.}

THEOREM 1. A quantum system $(L, M)$ has a dispersion-free state if and only if $L$ has an atom in its center.

Proof. To prove necessity, let $m$ be dispersion-free and let $L_{m}$ $=\{a \in L: m(a)=1\}$. Note that $L_{m} \neq \varnothing$ and let $T$ be a totally ordered subset of $L_{m}$. Now $a_{0}=\Lambda\{a \in T\}$ exists since the propositions in $T$ are mutually compatible and $m\left(a_{0}\right)=1$ by (M3), so $a_{0} \in L_{m}$ and $a_{0} \leqq a$ for all $a \in T$. By Zorn's lemma $L_{m}$ has a minimal element $a_{1}$. We first show $a_{1} \leqq a$ for all $a \in L_{m}$. Let $a_{2} \in L_{m}$. Now there is a nonzero proposition $a_{3}$ such that $a_{3} \leqq a_{1}, a_{2}$ since otherwise $a_{1} \wedge a_{2}=0$ and $m\left(a_{1} \wedge a_{2}\right)=0$ which contradicts (M2). Now there is a $b$ in $L$ such that $a_{3}+b=a_{1}$. If $m\left(a_{3}\right)=0$, then $m(b)=1$ and since $b \leqq a_{1}$ we have $b=a_{1}$. Hence $a_{3}=0$, a contradiction. Therefore $m\left(a_{3}\right)=1$ and again since $a_{3} \leqq a_{1}$, 
$a_{3}=a_{1}$ and thus $a_{1} \leqq a_{2}$. We now show that $a_{1} \in Z$. If $b \in L_{m}$, then $a_{1} \leqq b$ and $a_{1} \leftrightarrow b$. If $b \in L_{m}$ then $b^{\prime} \in L_{m}$ and $a_{1} \leqq b^{\prime}$. Thus $a_{1} \leftrightarrow b^{\prime}$ and it easily follows that $a_{1} \leftrightarrow b$. To show that $a_{1}$ is an atom, notice that $a_{1} \neq 0$ since $m\left(a_{1}\right)=1$ and suppose $b \leqq a_{1}$. If $b \in L_{m}$, then $b=a_{1}$. If $b \notin L_{m}$, then $b^{\prime} \in L_{m}$ and $a_{1} \leqq b^{\prime}$. Hence $b \leqq b^{\prime}$ and $b=b \wedge b \leqq b \wedge b^{\prime}=0$. To prove sufficiency let $a_{1} \in Z$ be an atom, let $m\left(a_{1}\right)=1$, and let $b \in L$. Since $b \leftrightarrow a_{1}, a_{1} \wedge b$ exists. Since $a_{1} \wedge b \leqq a_{1}$ we must have $a_{1} \wedge b=a_{1}$ or $a_{1} \wedge b=0$. If $a_{1} \wedge b=a_{1}$, then $a_{1} \leqq b$ and $m(b)=1$. If $a_{1} \wedge b=0$ it easily follows that $a_{1} \perp b$ and hence $m(b)=0$. Therefore $m$ is dispersion-free.

CoROLlary. A nontrivial coherent quantum system has no dispersionfree states.

Theorem 2. A logic $L$ admits hidden variables if and only if $L$ is atomic, complete, and deterministic.

Proof. If $L$ admits hidden variables, by our lemma there is a set of dispersion-free states $M$ satisfying (M1), (M2), and (M3). If $0 \neq a \in L$ then by (M1) there is a dispersion-free state $m$ such that $m(a)=1$ and by the proof of Theorem 1 there is an atom $a_{1}$ such that $a_{1} \leqq a$ and hence $L$ is atomic. Let $a \in L$ and let $\left\{a_{\alpha}: \alpha \in A\right\}$ be the atoms in $a$. By the proof of Theorem 1, the $a_{\alpha}$ 's are compatible and hence $c=V a_{\alpha}$ exists. If $c \neq a$ then there is a $b \neq 0$ such that $c+b=a$. But then $b$ contains an atom that $a$ does not which is a contradiction. Hence $a=\bigvee a_{\alpha}$. To show $L$ is a lattice, let $a, b \in L$, let $\left\{b_{\alpha}: \alpha \in A\right\}$ be the atoms in both $a$ and $b$, and let $c=\mathrm{V}_{\alpha}$. Now $c \leqq a, b$ and suppose $d \leqq a, b$. Since $d$ is the supremum of the atoms in $d$ we must have $d \leqq c$, hence $a \wedge b=c$, and $L$ is a lattice. We now show $L$ is a Boolean algebra. For $a, b, c \in L$ we must show that $a \wedge(b \bigvee c)=(a \wedge b) \bigvee(a \wedge c)$. Obviously $d=a \wedge(b \bigvee c) \geqq(a \wedge b) \bigvee(a \wedge c)=e$. Suppose $m \in M$ and $m(d)=1$. Now $m(a) \neq 0$. If $m(a)=m(b)=m(c)=1$ then $m(e)=1$. If $m(a)=1$, and $m(b)=m(c)=0$ we contradict (M2). If $m(a)=m(b)=1$, $m(c)=0$ then $m(e)=1$ and the last case $m(a)=m(c)=1, m(b)=0$ is the same. If $m(d)=0$, then obviously $m(e)=0$. Hence $m(d)=m(e)$ for all $m$ in $M$. Now there is an $f$ in $L$ such that $e+f=d$. It follows that $m(f)=0$ for all $m$ in $M$ and hence $f=0$ by (M1). Thus $e=d$ and $L$ is a Boolean algebra. It follows that $L$ is deterministic and hence complete. Conversely, suppose $L$ is atomic, complete and deterministic and let $\left\{a_{\alpha}: \alpha \in A\right\}$ be the atoms in $L$. Define the state $m_{\alpha}$ by $m_{\alpha}(a)=1$ if $a_{\alpha} \leqq a$ and $m(a)=0$ otherwise for all $\alpha$ in $A$. Then $m_{\alpha}$ is dispersion-free for all $\alpha$ in $A$. Letting $M=\left\{m_{\alpha}: \alpha \in A\right\}$ it easily follows that $M$ satisfies (M1), (M2), (M3) and by our lemma $L$ admits hidden variables. 
REMARKs. In Mackey's axiomatic model [6] it is assumed that the states are countably additive. Under this assumption our theorems and proofs still hold. Notice that our theorems and proofs still hold if in the definition of a quantum system we make the much weaker assumption that (M2) and (M3) hold only for dispersion-free states.

4. Physical interpretations. The ideal classical system is based on an $n$-dimensional Euclidean space $S$ called phase space, where the propositions $L$ are assumed to be the class of all subsets of $S$. The condition of the system is given by a point $p \in S$ and is described by a state $m$ concentrated at $p$, i.e., a finitely additive set function $m: L$ $\rightarrow[0,1]$ such that $m(p)=1$. We also permit mixtures of these states. These states are, of course, dispersion-free or mixtures of dispersionfree states, and conversely it is easily seen that dispersion-free and mixtures of dispersion-free states must be of this type. Thus the ideal classical system gives a model for a system which admits hidden variables. Physically, the important properties of the ideal classical system is that $L$ is atomic, deterministic, and complete.

It then follows from Theorem 2 that a quantum system admits hidden variables if and only if it acts physically as an ideal classical system. Now it is well known that quantum systems do not behave like classical systems (e.g. the Heisenberg uncertainty principle) [4] so hidden variables must be excluded from quantum mechanics. A coherent quantum system is one which physically has no "superselection rules" (cf. [14]). Theorem 1 tells us that such systems can not have any dispersion-free states, so again hidden variables are excluded.

5. Examples. We first give an example of a quantum system whose logic is not a lattice. This is a simplified version of an example in [10]. Let $L$ be the subsets of $\{1,2,3,4,5,6\}$ with an even number of elements together with $\varnothing$. $L$ is a logic under the usual order and complementation. However, $L$ is not a lattice since the infimum of $a_{1}=\{1,2,3,4\}$ and $a_{2}=\{2,3,4,5\}$ does not exist. This follows since $\{2,3\}$ and $\{3,4\}$ are both $\leqq a_{1}, a_{2}$ yet there is no proposition $c$ such that $c \leqq a_{1}, a_{2}$ and $\{2,3\},\{3,4\} \leqq c$. Let $m_{i j}, i, j=1, \cdots, 6, i \neq j$ be the state which satisfies $m_{i j}(a)=1$ if $i, j \in a, m_{i j}(a)=\frac{1}{2}$ if $i \in a, j \notin a$ or $j \in a, i \notin a$, and $m_{i j}(a)=0$ otherwise, $a \in L$. Let $M$ be the set of mixtures of the states $m_{i j}, i, j=1, \cdots, 6, i<j$. One can now check that ( $L, M)$ satisfies (M1), (M2), (M3) and is thus a quantum system.

Our next example shows that (M2) is necessary if our theorems are to hold. Let $L$ be the complemented lattice composed of distinct ele- 
ments $0,1, a, a^{\prime}, b, b^{\prime}$ in which the supremum of any two nonzero elements is 1 . Define the states $m_{1}, m_{2}$ by $m_{1}(a)=m_{1}(b)=m_{1}(1)=1$, $m_{2}\left(a^{\prime}\right)=m_{2}\left(b^{\prime}\right)=m_{2}(1)=1$. Then $m_{1}, m_{2}$ are dispersion-free. Since $m_{1}(a)=m_{1}(b)=1$, and $m_{1}(a \wedge b)=m_{1}(0)=0$, (M2) does not hold. Thus if $M$ is the set of mixtures of $m_{1}, m_{2},(L, M)$ has all the properties of a quantum system except (M2). Since $M$ is a mixture of dispersionfree states and there are not any atoms in the center Theorems 1 and 2 do not hold.

6. Comments. Recently a large number of papers have appeared concerning hidden variables, both in the mathematical and physical literature. Besides the papers already mentioned, in the physical literature the reader might consult $[1],[2],[3],[9],[12],[13]$ where [3] has a fairly complete bibliography of other relevant papers while in the mathematical literature the reader might consider [5], [11], [15]. We shall briefly compare our work to that in [5] and [15].

In [5] a necessary condition for the existence of hidden variables is derived (Theorem 0 or better yet its counterpart after the definition on p. 84) which is essentially the same as our criteria. Then an example of a finite quantum system $D$ (called a partial Boolean algebra in this paper) is given which does not admit even one dispersion-free state (here called homomorphisms onto $Z_{2}$ ). $D$ is a certain set of linear subspaces of $R^{3}$ and its importance lies in the fact that it corresponds to the propositions of an actual concrete quantum mechanical experiment. Our considerations are more general since we are concerned with abstract quantum systems and not just finite systems of linear subspaces. In fact for finite systems our proofs hold for any partial Boolean algebra, since in the finite case we automatically have completeness with respect to compatible elements and Axiom (M3) becomes redundant. Also it is trivial to check that Axiom (M2) holds for logics of subspaces.

Comparing our work to that of [15] our definition of the existence of hidden variables is essentially equivalent to the existence of an additive embedding of $L$ in to a Boolean algebra. In [15] an example is given (the lattice of closed subspaces of a Hilbert space) which shows that additive embeddings need not exist. Our results are much stronger. We have shown that $L$ can never be additively embedded in a Boolean algebra unless $L$ itself is a Boolean algebra.

It should be noted that to get our stronger results we have imposed additional conditions on our dispersion-free states namely axioms (M2) and (M3). Thus our dispersion-free states are different than the homomorphisms onto $Z_{2}$ in [5] or the two-valued states in [15]. 


\section{REFERENCES}

1. J. Bell, On the problem of hidden variables in quantum mechanics, Rev. Modern Phys. 38 (1966), 447-452.

2. D. Bohm and J. Bub, $A$ proposed solution to the measurement problem in quantum mechanics by hidden variables, Rev. Modern Phys. 38 (1966), 453-469.

3. —_- A refutation of the proof by Jauch and Piron that hidden variables can be excluded in quantum mechanics, Rev. Modern Phys. 38 (1966), 470-485.

4. J. Jauch and C. Piron, Can hidden variables be excluded in quantum mechanics?, Helv. Phys. Acta 36 (1963), 827-837.

5. S. Kochen and E. Specker, The problem of hidden variables in quantum mechanics, J. Math. Mech. 17 (1967), 59-87.

6. G. Mackey, The mathematical foundations of quantum mechanics, Benjamin, New York, 1963.

7. M. Mac Laren, Notes on axioms for quantum mechanics, ANL-7065, Argonne National Laboratory, 1965.

8. J. von Neumann, Mathematical foundations of quantum mechanics, Princeton Univ. Press, Princeton, N. J., 1955.

9. C. Papaliolious, Experimental test of a hidden variable quantum theory, Phys. Rev. Lett. 18 (1967), 622-625.

10. A. Ramsey, A theorem on two commuting observables, J. Math. Mech. 15 (1966), 227-234.

11. B. Rankin (Editor), Differential space, quantum systems and prediction, M.I.T. Press, Cambridge, Mass., 1966.

12. J. Tutsch, Collapse time for the Bohm-Bub hidden variable theory, Rev. Modern Phys. (to appear).

13. R. Wagsness, Hidden variables and magnetic relaxation, Phys. Rev. (to appear).

14. G. Wick, E. Wigner and A. Wightman, Intrinsic parity of elementary particles, Phys. Rev. 88 (1952), 101-105.

15. N. Zierler and M. Schlessinger, Boolean embeddings of orthomodular sets and quantum logic, Duke Math. J. 32 (1965) 251-262.

UNIVERSITY OF WISCONSIN 